Applied Mathematical Sciences, Vol. 9, 2015, no. 12, 579 - 582

HIKARI Ltd, www.m-hikari.com

http://dx.doi.org/10.12988/ams.2015.411977

\title{
Poisson Approximation for a Sum of Independent Yule Random Variables
}

\author{
K. Teerapabolarn \\ Department of Mathematics, Faculty of Science \\ Burapha University, Chonburi 20131, Thailand
}

Copyright (c) $2014 \mathrm{~K}$. Teerapabolarn. This is an open access article distributed under the Creative Commons Attribution License, which permits unrestricted use, distribution, and reproduction in any medium, provided the original work is properly cited.

\begin{abstract}
This paper uses the Stein-Chen method and the Yule $w$-functions to determine a bound for the total variation distance between the distribution of a sum of independent Yule random variables and a Poisson distribution with mean $\sum_{i=1}^{n} \frac{1}{a_{i}-1}$, where $a_{i}$ is parameter of each Yule distribution. With the bound, it is indicated that the distribution of that summands can be approximated by a Poisson distribution with this mean when all $a_{i}$ are large.
\end{abstract}

Mathematics Subject Classification: 62E17, 60F05, 60G50

Keywords: Poisson approximation, Yule distribution, Stein-Chen method, $w$-functions

\section{Introduction}

Let $X_{1}, \ldots, X_{n}$ be independently distributed Yule random variables, each with probability $P\left(X_{i}=k\right)=\frac{a_{i} k ! \Gamma\left(a_{i}+1\right)}{\Gamma\left(a_{i}+k+2\right)}, k \in \mathbb{N} \cup\{0\}$, mean $\mu_{i}=\frac{1}{a_{i}-1}$ and variance $\sigma_{i}^{2}=\frac{a_{i}^{2}}{\left(a_{i}-2\right)\left(a_{i}-1\right)^{2}}$, where $a_{i}>2$. Let $\mathcal{Y}_{n}=\sum_{i=1}^{n} X_{i}$ and $\mathcal{P}_{\lambda_{n}}$ denote the the Poisson random variable with mean $\lambda_{n}=\sum_{i=1}^{n} \mu_{i}$. For $n=1$, Teerapabolarn [2] gave a bound for the total variation distance between a Yule distribution with parameter $a_{1}>2$ and a Poisson distribution with mean $\lambda_{1}=\frac{1}{a_{1}-1}$ as follows:

$$
d_{T V}\left(\mathcal{Y}_{1}, \mathcal{P}_{\lambda_{1}}\right) \leq \frac{3 a_{1}-1}{\left(a_{1}^{2}-1\right)\left(a_{1}-1\right)}
$$


where $d_{T V}\left(\mathcal{Y}_{1}, \mathcal{P}_{\lambda_{1}}\right)=\sup _{A \subseteq \mathbb{N} \cup\{0\}}\left|P\left(\mathcal{Y}_{1} \in A\right)-P\left(\mathcal{P}_{\lambda_{1}} \in A\right)\right|$.

In this paper, we are interested to determine a bound for approximating the distribution of a sum of $n(>1)$ independent Yule random variables by a Poisson distribution with mean $\lambda_{n}$, in the form of $d_{T V}\left(\mathcal{Y}_{n}, \mathcal{P}_{\lambda_{n}}\right)$. The tools for giving the desired result are the Stein-Chen method and $w$-functions, which are in Section 2. In Section 3, our result is derived by these tools and the conclusion of this study is presented in the last section.

\section{Method}

The following lemma is need to prove the desired result, which is directly obtained from $[2]$.

Lemma 2.1. For $1 \leq i \leq n$, let $w_{i}$ be the $w$-function associated with the Yule random variable $X_{i}$, then we have the following:

$$
w_{i}(k)=\frac{(k+1)^{2}}{\left(a_{i}-1\right) \sigma_{i}^{2}}, k=0,1, \ldots
$$

For the Stein-Chen method, following [1], it is applied for every constant $\lambda_{n}>0$, every subset $A$ of $\mathbb{N} \cup\{0\}$ and the bounded real valued function $f=f_{A}: \mathbb{N} \cup\{0\} \rightarrow \mathbb{R}$. Thus, Stein's equation for the Poisson distribution with mean $\lambda_{n}$ is of the form

$$
P\left(\mathcal{Y}_{n} \in A\right)-P\left(\mathcal{P}_{\lambda_{n}} \in A\right)=\mathbb{E}\left[\lambda_{n} f\left(\mathcal{Y}_{n}+1\right)-\mathcal{Y}_{n} f\left(\mathcal{Y}_{n}\right)\right]
$$

For any subset $A$ of $\mathbb{N} \cup\{0\}$ and for every $x \in \mathbb{N}$, Barbour et al. [1] showed that

$$
\sup _{A}|\Delta f(k)|=\sup _{A}|f(k+1)-f(k)| \leq \frac{1}{k} .
$$

\section{Result}

The following theorem gives a bound on the error of Poisson approximation to the distribution of $\mathcal{Y}_{n}$.

Theorem 3.1. With the above definitions, the following inequality holds:

$$
d_{T V}\left(\mathcal{Y}_{n}, \mathcal{P}_{\lambda_{n}}\right) \leq \sum_{i=1}^{n} \frac{3 a_{i}-1}{\left(a_{i}^{2}-1\right)\left(a_{i}-1\right)}
$$


Proof. From (2.2), we have that

$$
\begin{aligned}
d_{T V}\left(\mathcal{Y}_{n}, \mathcal{P}_{\lambda_{n}}\right) & =\left|\mathbb{E}\left[\lambda_{n} f\left(\mathcal{Y}_{n}+1\right)-\mathcal{Y}_{n} f\left(\mathcal{Y}_{n}\right)\right]\right| \\
& \leq \sum_{i=1}^{n} \mathbb{E}\left\{\left|\mu_{i}-\sigma_{i}^{2} w_{i}\left(X_{i}\right)\right||\Delta f(X)|\right\} \quad \text { by [3]) } \\
& \leq \sum_{i=1}^{n} \sum_{k=1}^{\infty}\left|\mu_{i}-\sigma_{i}^{2} w_{i}(k)\right| \sup _{A}|\Delta f(k)| P\left(X_{i}=k\right) \\
& \left.\leq \sum_{i=1}^{n} \sum_{k=1}^{\infty}\left|\frac{1}{a_{i}-1}-\frac{(k+1)^{2}}{a_{i}-1}\right| \frac{1}{k} P\left(X_{i}=k\right) \quad \text { by }(2.1) \text { and }(2.3)\right) \\
& \leq \sum_{i=1}^{n} \sum_{k=1}^{\infty}\left|-\frac{k(k+2)}{a_{i}-1}\right| \frac{1}{k} P\left(X_{i}=k\right) \\
& =\sum_{i=1}^{n} \sum_{k=1}^{\infty} \frac{k+2}{a_{i}-1} P\left(X_{i}=k\right) \\
& =\sum_{i=1}^{n} \frac{\mu_{i}+2\left(1-P\left(X_{i}=0\right)\right)}{a_{i}-1} \\
& =\sum_{i=1}^{n} \frac{3 a_{i}-1}{\left(a_{i}^{2}-1\right)\left(a_{i}-1\right)}
\end{aligned}
$$

which yields the result in (3.1).

When all $X_{i}$ are identically distributed random variables, thus immediately from the Theorem 3.1, we have the following Corollary.

Corollary 3.1. If $a_{1}=\cdots=a_{n}=a$, then $\lambda_{n}=\frac{n}{a-1}$ and we have the following:

$$
d_{T V}\left(\mathcal{Y}_{n}, \mathcal{P}_{\lambda_{n}}\right) \leq \frac{(3 a-1) n}{\left(a^{2}-1\right)(a-1)}
$$

\section{Conclusion}

In this study, a bound on the total variation distance between the distribution of a sum of independent Yule random variables and a Poisson distribution was obtained by using the Stein-Chen method and the Yule $w$-functions. With this bound, it is found that the distribution of the summands can be approximated by the Poisson distribution with mean $\lambda_{n}=\sum_{i=1}^{n} \frac{1}{a_{i}-1}$ when all $a_{i}$ are large. 


\section{References}

[1] A.D. Barbour, L. Holst, S. Janson, Poisson approximation, Oxford Studies in Probability 2, Clarendon Press, Oxford, 1992.

[2] K. Teerapabolarn, Bounds on approximating the Yule distribution by the Poisson and geometric distributions, Int. J. Appl. Math. Stat., 14 (2009), 86-93.

[3] K. Teerapabolarn, An extension of Poisson approximation by $w$-functions, Int. J. Pure Appl. Math., 87 (2013), 529-534. http://dx.doi.org/10.12732/ijpam.v87i4.3

Received: December 3, 2014; Published: January 7, 2015 